\title{
Peripheral eosinophilia as an indicator of meningitic angiostrongyliasis in exposed individuals
}

\author{
Kittisak Sawanyawisuth ${ }^{1,4} /{ }^{+}$, Kanlayanee Sawanyawisuth², Vichai Senthong', \\ Panita Limpawattana', Pewpan M Intapan ${ }^{3,4}$, Somsak Tiamkao', Suthipun Jitpimolmard', \\ Verajit Chotmongkol ${ }^{1,4}$, Elizabeth Barrett-Connor ${ }^{5}$
}

${ }^{1}$ Department of Medicine ${ }^{2}$ Department of Biochemistry ${ }^{3}$ Department of Parasitology, Faculty of Medicine ${ }^{4}$ The Research and Diagnostic
Centre for Emerging Infectious Disease, Khon Kaen University, 123 Mitraparp Friendship Rd., 40002 Khon Kaen, Thailand
${ }^{5}$ Department of Family and Preventive Medicine, School of Medicine, University of California, La Jolla, San Diego, CA, USA

The diagnosis of meningitic angiostrongyliasis (MA) is based on clinical criteria. A lumbar puncture is used as a diagnostic tool, but it is an invasive procedure. The blood eosinophil levels are also assessed and used in the diagnosis of this disease. We enrolled 47 patients with serologically proven MA and 131 controls with intestinal parasite infections. An absolute eosinophil count model was found to be the best marker for MA. An eosinophil count of more than 798 cells led to sensitivity, specificity, positive predictive and negative predictive values of $76.6 \%, 80.2 \%$, $58.1 \%$ and $90.5 \%$, respectively. These data support the use of testing for high blood eosinophil levels as a diagnostic tool for MA in individuals that are at risk for this disease.

Key words: eosinophilia - diagnosis - meningitis - angiostrongyliasis - Angiostrongylus cantonensis

Angiostrongylus cantonensis is the main pathogen that causes eosinophilic meningitis in humans (Chotmongkol et al. 2000). This condition is clinically diagnosed by the presence of eosinophils in the cerebrospinal fluid of a patient that has a history of eating raw freshwater snails, contaminated vegetables or other paratenic hosts such as shrimp, frogs or monitor lizards.

In endemic areas, meningitic angiostrongyliasis is suspected in patients presenting with an acute, severe headache and potential exposure to A. cantonensis larva. Eosinophils in the spinal fluid lead to an immediate presumptive diagnosis, but a lumbar puncture is invasive and expensive. A definite diagnosis after finding larva in the cerebrospinal fluid is extremely rare (Wang et al. 2008). The identification of additional laboratory indicators, such as white blood cell counts, that indicate the presence of peripheral eosinophilia would improve the clinical diagnosis process for meningitic angiostrongyliasis.

An increase in blood eosinophils has been shown to be a major effect of parasitic infection, particularly for tissue parasites such as A. cantonensis. Blood eosinophilia has been observed in $30-80 \%$ of patients with angiostrongyliasis (Chotmongkol et al. 2000, Jitpimolmard et al. 2007). The aim of the present study was to

Financial support: Research and Diagnostic Center for Emerging Infectious Disease, Khon Kaen University, Office of Higher Education Commission/Ministry of Education

+ Corresponding author: kittisak@kku.ac.th

Received 1 July 2010

Accepted 13 September 2010 evaluate the utility of blood eosinophils as a diagnostic tool for meningitic angiostrongyliasis. We compared the blood eosinophil levels in patients with serologically positive meningitic angiostrongyliasis to control patients with intestinal parasitic infections.

We recruited patients who were diagnosed with meningitic angiostrongyliasis at Srinagarind Hospital in Khon Kaen, Thailand, between 1996-2007. The inclusion criteria were the following: (i) a cerebrospinal fluid white blood cell count of 10 cells $/ \mathrm{mm}^{3}$ or more and the presence of 10 or more eosinophils $/ \mathrm{mm}^{3}$ in the cerebrospinal fluid, (ii) a value of at least $10 \%$ for the percentage of eosinophils in the cerebrospinal fluid leukocyte count, (iii) negative cerebrospinal fluid test results for organisms, cryptococcal antigens and cultures, (iv) a history of exposure to A. cantonensis and (v) a positive result on a test for the presence of serum antibodies to the $29 \mathrm{kDa}$ antigenic polypeptide of A. cantonensis (Maleewong et al. 2001). The control group consisted of adults who were infected by intestinal parasites and had received complete blood count test results between January 1-December 312007.

We retrospectively reviewed the baseline characteristics, symptoms, signs, stool examination results and the results of a complete blood count test of the patients. Clinical evidence of meningitis is defined as a combination of headache, fever and neck stiffness. The complete blood counts were composed of the white blood cell count (cells $/ \mathrm{mm}^{3}$ ), percent blood eosinophils (\%) and eosinophil count. The eosinophil count was defined as the (white blood cell x percent eosinophils)/100.

The baseline and clinical characteristics of the meningitic angiostrongyliasis cases and the controls were compared using descriptive statistics. The Wilcoxon rank sum test or a Student's $t$ test was used to compare the differences in the numerical variables where appropriate. 
The total number of white blood cells, the eosinophil count and the percent eosinophils were compared between the meningitic angiostrongyliasis cases and the control groups and were fitted by a logistic regression model to assess the probability of the patient having angiostrongyliasis.

Analyses to determine the best cut-off point, sensitivity, specificity, positive predictive value (PPV) and negative predictive value (NPV) were conducted for the optimal statistical parameters. All of the analyses were performed using SAS software version 8.2 on a personal computer.

The study protocol was reviewed and approved by the institutional review board and the Ethical Committee of Khon Kaen University.

There were 75 consecutive patients who were clinically diagnosed with meningitic angiostrongyliasis. These patients underwent a serologic test for $A$. cantonensis during the period between 1996-2007. Out of these patients, $47(62.7 \%)$ had positive results on a serologic test. The control group was comprised of 131 patients who also had a white blood cell count with the eosinophils enumerated. Men were the majority in both groups $(66 \%$ and $80 \%$ of the meningitic angiostrongyliasis cases and controls, respectively). The median age of the patients in the meningitic angiostrongyliasis group was 35 years (with a range of 15-62 years) and the mean age of the patients in the control group was also 35 years (with a range of 16-53 years). All of the meningitic angiostrongyliasis cases presented with headache. A stiff neck, fever and clinical evi- dence of meningitis were observed in $22(47 \%), 11(23 \%)$ and four $(9 \%)$ of the patients, respectively.

Of the 131 control patients, Strongyloides stercoralis was the most commonly observed parasite, which was observed in 73 of the control patients. A minute intestinal fluke infection, echinostomiasis, a hookworm infection, opisthrochiasis, trichomoniasis, taeniasis and giardiasis were observed one, four, seven, eight, nine, 12 and 17 of the subjects, respectively.

The white blood cell count, percent eosinophils and eosinophil count were significantly higher in the angiostrogyliasis group compared to the control group (Table I). The cut-point of an eosinophil count of 798 gave the best sensitivity, specificity and PPV and NPV of $76.6 \%, 80.2 \%$, $58.1 \%$ and $90.5 \%$, respectively (Table II). A white blood cell count of 10,600 cells $/ \mathrm{mm}^{3}$ or $9 \%$ eosinophils also led to the same specificity as the eosinophil count. However, the sensitivities of the white blood cells and the percent eosinophils were lower, at $44.7 \%$ and $74.5 \%$, respectively (Table II).

Eosinophils are granulocytes that are typically associated with immune responses to helminth infections. Even though the specific function of eosinophils in parasitic infections remains unclear (Rothenberg \& Hogan 2006), blood eosinophils levels have been shown in the present study to play a role as a rapid laboratory indicator of suspected meningitic angiostrongyliasis.

Meningitic angiostrongyliasis can be diagnosed using clinical criteria (Chotmongkol et al. 2000, Jitpimolmard et al. 2007, Ramirez-Avila et al. 2009), including a percentage of cerebrospinal fluid eosinophils that is greater than

TABLE I

Comparison of blood test findings between patients with serologically positive angiostrongyliasis and control group

\begin{tabular}{lccc}
\hline Variables & $\begin{array}{c}\text { Angiostrongyliasis } \\
(\mathrm{n}=47)\end{array}$ & $\begin{array}{c}\text { Controls } \\
(\mathrm{n}=131)\end{array}$ & $\mathrm{p}$ value \\
\hline White blood cell (cells $\left./ \mathrm{mm}^{3}\right)$ & 10,200 & 9,000 & $<0.0001$ \\
& $(6,600-17,400)$ & $(4,800-15,000)$ & $<0.0001$ \\
Percent eosinophils (\%) & $15(1-43)$ & $5(0.5-20.6)$ & $<0.0001$ \\
Eosinophils count (cells) & $1,632(142-6,192)$ & $456(31-2,293)$ & $<$ \\
\hline
\end{tabular}

data reported as median (range), eosinophils count equals (percent eosinophils x total white blood cell)/100.

TABLE II

Statistical parameters of various chosen cut-points for the diagnosis of meningitic angiostrongyliasis

\begin{tabular}{|c|c|c|c|c|}
\hline Cut-points & Sensitivity & Specificity & $\begin{array}{c}\text { Positive } \\
\text { predictive value }\end{array}$ & $\begin{array}{c}\text { Negative } \\
\text { predictive value }\end{array}$ \\
\hline White blood cell 10,600 cells $/ \mathrm{mm}^{3}$ & 44.7 & 80.2 & 44.7 & 80.2 \\
\hline $9 \%$ eosinophils & 74.5 & 80.2 & 57.4 & 89.7 \\
\hline Eosinophils count of 798 cells & 76.6 & 80.2 & 58.1 & 90.5 \\
\hline
\end{tabular}

eosinophils count equals (percent eosinophils x total white blood cell)/100. 
$10 \%$ and a history of eating raw fresh-water snails or other transmission hosts. Our serologic test is very specific for meningitic angiostrongyliasis (99\% specificity), but the sensitivity is $55.6 \%$. In contrast, the eosinophil count of 798 cells was sensitive and specific for meningitic angiostrongyliasis in $80 \%$ of cases (Table II).

In addition to good sensitivity, both the percentage of eosinophils and the eosinophil count cut-points had a very high NPV for meningitic angiostrongyliasis (Table II). These findings indicate that if a patient has a percent eosinophils or eosinophil count less than $9 \%$ or 798 cells, respectively, the likelihood of the patient having angiostrongyliasis is low.

Every patient with meningitis requires a lumbar puncture. Unlike in bacterial meningitis, clinical evidence of meningitis is uncommon in meningitic angiostrongyliasis. Based on the results of the present study and a previous study (Chotmongkol et al. 2000), clinical evidence of meningitis was found in only $9-10 \%$ of the meningitic angiostrongyliasis patients. As a result, clinicians may easily miss this condition during diagnosis. A delayed diagnosis or the misdiagnosis of meningitic angiostrongyliasis may increase the risk of developing the encephalitic form of the disease, which has high morbidity and mortality (Sawanyawisuth et al. 2009). As a result, we suggest that testing the level of blood eosinophils should be a leading tool in the diagnosis of meningitic angiostrongyliasis. Additionally, clinicians using this test can be more confident in their diagnosis prior to performing lumbar puncture.

There are two main limitations in the present study. First, other tissue parasites may induce extremely high levels of blood eosinophils, such as gnathostomiasis or toxocariasis. Even though the clinical features of both of these conditions are different from those for angiostrongyliasis (Ramirez-Avila et al. 2009), the suggested cut-points should be only used for suspected meningitic angiostrongyliasis. An acute headache and a history of exposure to $A$. cantonensis larva are important clues for the diagnosis of meningitic angiostrongyliasis. Second, even though the blood eosinophil level may not reach the cut-points in some patients, a lumbar puncture may still be needed if clinical signs such as fever or neck stiffness are present. Also, a lumbar puncture has been shown to be an acute reliever of headache in addition to its diagnostic role (Sawanyawisuth et al. 2004).
In a previous study, all of the meningitic angiostrongyliasis cases were shown to have positive serologic test results (Maleewong et al. 2001). This test was done using an immunoblot analysis based on the reaction of specific $\mathrm{IgG}$ antibodies reaction to the $29 \mathrm{kDa}$ antigenic polypeptide of $A$. cantonensis. In addition, this test had a specificity value of $99.4 \%$. In most locations, this test result cannot be provided on the same day that the patient presents and this test is most useful for research because it is not yet widely available.

In summary, high blood eosinophil levels appear to be a rapid, inexpensive and widely available tool for the diagnosis of meningitic angiostrongyliasis in symptomatic patients.

\section{REFERENCES}

Chotmongkol V, Sawanyawisuth K, Thavornpitak Y 2000. Corticosteroid treatment of eosinophilic meningitis. Clin Infect Dis 31: 660-662.

Jitpimolmard S, Sawanyawisuth K, Morakote N, Vejjajiva A, Puntumetakul M, Sanchaisuriya K, Tassaneeyakul W, Tassaneeyakul W, Korwanich N 2007. Albendazole therapy for eosinophilic meningitis caused by Angiostrongylus cantonensis. Parasitol Res 100: 1293-1296.

Maleewong W, Sombatsawat P, Intapan PM, Wongkham C, Chotmongkol V 2001. Immunoblot evaluation of the specificity of the 29-kDa antigen from young adult female worms Angiostrongylus cantonensis for immunodiagnosis of human angiostrongyliasis. Asian Pac J Allergy Immunol 19: 267-273.

Ramirez-Avila L, Slome S, Schuster FL, Gavali S, Schantz PM, Sejvar J, Glaser CA 2009. Eosinophilic meningitis due to Angiostrongylus and Gnathostoma species. Clin Infect Dis 48: 322-327.

Rothenberg ME, Hogan SP 2006. The eosinophil. Annu Rev Immunol 24: $147-174$.

Sawanyawisuth K, Limpawattana P, Busaracome P, Ninpaitoon B, Chotmongkol V, Intapan PM, Tanawirattananit S 2004. A 1-week course of corticosteroids in the treatment of eosinophilic meningitis. Am J Med 117: 802-803.

Sawanyawisuth K, Takahashi K, Hoshuyama T, Sawanyawisuth K, Senthong V, Limpawattana P, Intapan PM, Wilson D, Tiamkao S, Jitpimolmard S, Chotmongkol V 2009. Clinical factors predictive of encephalitis caused by Angiostrongylus cantonensis. Am J Trop Med Hyg 81: 698-701.

Wang QP, Lai DH, Zhu XQ, Chen XG, Lun ZR 2008. Human angiostrongyliasis. Lancet Infect Dis 8: 621-630. 